\title{
Bayesian detection of planetary transits
}

\section{A modified version of the Gregory-Loredo method for Bayesian periodic signal detection}

\begin{abstract}
S. Aigrain ${ }^{\star}$ and F. Favata
Astrophysics Division - Space Science Department of ESA, ESTEC, Postbus 299, 2200 AG Noordwijk, The Netherlands e-mail: Fabio.Favata@rssd.esa.int

Received 17 December 2001 / Accepted 3 September 2002

Abstract. The detection of planetary transits in stellar photometric light-curves is poised to become the main method for finding substantial numbers of terrestrial planets. The French-European mission COROT (foreseen for launch in 2005) will perform the first search on a limited number of stars, and larger missions Eddington (from ESA) and Kepler (from NASA) are planned for launch in 2007. Transit signals from terrestrial planets are small $\left(\Delta F / F \simeq 10^{-4}\right)$, short $(\Delta t \simeq 10$ hours) dips, which repeat with periodicity of a few months, in time series lasting up to a few years. The reliable and automated detection of such signals in large numbers of light curves affected by different sources of noise is a statistical and computational challenge. We present a novel algorithm based on a Bayesian approach. The algorithm is based on the Gregory-Loredo method originally developed for the detection of pulsars in X-ray data. In the present paper the algorithm is presented, and its performance on simulated data sets dominated by photon noise is explored. In an upcoming paper the influence of additional noise sources (such as stellar activity) will be discussed.
\end{abstract}

Key words. planetary systems - occultations - methods: data analysis

\section{Introduction}

The search for rocky, terrestrial planets around other stars is a key research topic in astrophysics for the next decade. Following the first exo-planet detection around a sun-like star (Mayor \& Queloz 1995), gaseous giants around other solartype stars have been shown to be relatively common (Butler et al. 2001). The mass function of the current crop of extrasolar planets grows rapidly toward the lower masses (Butler et al. 2001), showing that low-mass planets must be common. However, the radial velocity technique, which has resulted in the detection of the exo-planets detected so far, is limited to planetary masses somewhat smaller than Saturn, and cannot reach the domain of terrestrial planets. This is due to astrophysical effects, such as microturbulence in the star's atmosphere, rather than instrumental limitations.

The most promising approach for the detection of (significant numbers) of terrestrial planets around stars other than the Sun appears to be the search for planetary transits, i.e. dips in the light curve of the parent stars caused by the planet transiting in front of the stellar disk. The flux dip caused

Send offprint requests to: S. Aigrain, e-mail: suz@ast.cam.ac.uk

* Now at IoA - Madingley Road, Cambridge CB3 OHA, UK. by the transit is also small, $\Delta F / F=\left(R_{\mathrm{p}} / R_{*}\right)^{2}$, which for the transit of an Earth-Sun system gives $\Delta F / F=10^{-4}$. This is well below the scintillation noise caused by the Earth's atmosphere (Favata \& the Eddington Science Team 2000, see), so that highaccuracy space-based photometry will be needed for the detection of such events. The probability of occurrence of a transit depends on the inclination of the planetary orbit relative to the line of sight (which must be close to $i=90$ degrees), and is relatively small (for a set of randomly oriented Sun-Earth systems $p \simeq 0.5 \%$ ), so that searches for planetary transits must be based on observation of large samples of target stars. A typical transit duration will be of order $\Delta t \simeq 10$ hours, and the transit periodicity will be the same as the orbital period of the planet, typically several months.

A number of space missions wholly or partially dedicated to the search for planetary transits are either in development or in the planning stage. The CNES/European satellite COROT is planned for launch in 2005, while the ESA mission Eddington and the NASA mission Kepler are planned for launch in 2007. Given the intrinsically statistical nature of planetary transit searches, these missions will acquire large number of stellar light curves, ranging from thousands for COROT to hundreds of thousands for Eddington and Kepler. Also, some smaller 
searches are being conducted for limited time periods (and concentrating on larger planets) using e.g. HST (Gilliland et al. 2000) or ground-based telescopes (e.g. Doyle et al. 2000).

The analysis of data from such searches, and in particular the detection of transits with a high degree of certainty and a low false alarm rate, is a challenging task. The transit signal is weak $\left(\Delta F / F=10^{-4}\right)$, and concentrated in a small fraction of the total signal: for a habitable planet orbiting a $\mathrm{K} 5 \mathrm{~V}$ star the orbital period will be roughly 4 months, so that for a 1 year light curve three events will be present. As each transit lasts $\approx 10$ hours, the transit signal is present in only $\approx 0.3 \%$ of the total light curve. In the Euclidean regime, the number of stars in a given field increases toward fainter magnitudes by a factor of $\approx 4$ per magnitude. This is the case for the range of magnitudes and the low Galactic target latitudes of interest for currently planned missions. Therefore, most of the detected planets will be in the light curves of the fainter (and thus statistically noisier) stars, impying the need for effective robust data analysis algorithms able to reliably detect transits "hidden in the noise". At the same time, the large number of light curves which will need to be analyzed, each with a large number of points (of order 10000 points for a year of data) makes the use of efficient algorithms necessary, and rules out brute force approaches.

Some ground- (Doyle et al. 2000) and HST-based (Gilliland et al. 2000) transit searches, which deal with relatively small numbers of light curves, use a detection approach based on comparing large numbers of model transits to the light curves and minimising a $\chi^{2}$ statistic (or a linear statistic in the case of Doyle). These approaches are computationally very intensive, and thus may be unsuitable for the routine processing of the large number of light curves which will be produced by upcoming space missions.

As an alternative, transit detection algorithms based on Bayesian methods have recently been the subject of some attention. They have the advantage of combining computational efficiency with flexibility. While a global statistic can be used for the detection, information is directly available to reconstruct the detected signal if wanted, therefore providing a tool to discriminate between planetary transits and other types of periodic signals (Defaÿ et al. 2001a), as well as directly measuring additional planetary characteristics such as the planet's radius.

In the present paper we present a novel algorithm for the detection of planetary transits based on the method developed by Gregory \& Loredo (1992) (hereafter referred to as GL method) for the search of pulsed emission from pulsars in $\mathrm{X}$-ray data. While the algorithm was developed to be "general purpose", we have tuned it with the parameters of the upcoming Eddington planet finding mission in mind. The present paper discusses the characteristics of the algorithm on the basis of extensive simulations for the case in which the light curve is dominated by photon noise. Its performance in the case in which stellar activity is the dominating noise source will be the subject of a future paper.

Bayesian algorithms for the detection of planetary transits are also being developed in the context of the COROT mission. In particular, an approach based on expansion of the light curve into a truncated Fourier series is being investigated (Defaÿ et al. 2001b). Perfoming the detection in the Fourier domain can make the algorithm computationally sensitive to data gaps and sampling rates. Here we explore a more robust direct space approach.

The GL (Gregory \& Loredo 1992) method, was initially developed for the detection of X-ray pulsars (where Poisson statistics dominate) and later extended to the Gaussian noise case (Gregory 1999). At the flux levels of interest for the transit searches for Eddington, the photon shot noise per detection element (which is Poissonian) can be very well represented by Gaussian noise (see Sect. 2.1). The original formulation of the GL algorithm is well-suited to the detection of periodic signals of unknown shape. However, in the planetary transit problem we have strong prior information about the transit shape. In this paper we modify the GL algorithm to perform more optimally for planetary transit detection. We do this by allowing one of the bins to have a variable width, to represent the out of transit constant signal level. This formulation also permits the phase of the transits to be identified, a task the original GL method is not suited for (see Sect. 3.1). The fitted parameters are the period, duration and phase of the transit. The shape of the transit can then be reconstructed from the phase-folded light curve.

The simulated light curves are described in Sect. 2. The algorithm is outlined in Sect. 3 and compared with the original GL algorithm in Sect. 4. Section 5 describes the evaluation of the algorithm's performance by determining the number of false alarms and missed detections in a large sample of simulated light curves with and without transits. Conclusions and options for future work are presented in Sect. 7.

\section{The light curves}

\subsection{Transits}

Given the presence of limb darkening in stellar photospheres, planetary transits are not perfectly "flat bottomed" (nor are they, strictly speaking, truly grey). To simulate transits in a realistic way, the Universal Transit Modeler (UTM) software written by H. J. Deeg (Deeg 1999) was used. Limb darkening coefficients were taken from Van Hamme (1993). Two types of transits were simulated, one representing a Jupiter-type planet in a short orbit around a Sun-like star and another representing a Earth-like planet in an habitable orbit around a K5V star.

The input characteristics of the system for the Jovian transit were:

- Time step $t_{\text {unit }}=15 \mathrm{~min}$.

- Radius of star $R_{*}=R_{\odot}$.

- Luminosity of star $L_{*}=L_{\odot}$.

- Radius of planet $R_{\mathrm{p}}=R_{4}$. The ratio of the duration of the ingress/egress, to that of the "flat bottom" of the transit (affected only by limb darkening) is roughly $2 R_{\mathrm{p}} /\left(R_{\star}-R_{\mathrm{p}}\right)$, in this case $\simeq 0.22$.

- Period of transit $P=2880 \times t_{\text {unit }}$ (1 month). This is at the short end of the range of periods of interest for Eddington, but extrapolation to longer periods is to some extent possible (when activity is not included) by acting on the number of transits in the light curve.

- The distance star-planet, which is used to determine the transit duration, was varied between $d=15.3 \times R_{\odot}$ 
(resulting in a transit duration of 15 hours) and $d=45.9 \times$ $R_{\odot}$ (5 hours). (N.B.: for the same period different distances would correspond to different planetary to stellar mass ratios).

- The duration of light curve was varied between $D=3 \times P$ and $D=5 \times P$.

- The phase of the transit was randomly varied in the different simulations. The posital phase (between 0 and 1) is used in the course of the present paper.

Light curves were normalized to the photon count level expected for a star of a given $V$ magnitude (between 7 and 17 typically), based on the throughput expected for the baseline Eddington mission design (Favata \& the Eddington Science Team 2000), i.e. a collecting area of $0.6 \mathrm{~m}^{2}$ and a total system throughput of $70 \%$. With these instrument parameters a $V=21.5 \mathrm{G} 2 \mathrm{~V}$ star will yield $\simeq 50$ detected photons/s. Gaussian noise was then added with variance defined by the number of detected photons per pixel.

\section{The algorithm}

\subsection{A Bayesian method}

The method employed consists of calculating the likelihood of the data given a certain number of parameters, varying the parameters over a given range and identifying the value of each parameter whose probability is maximized according to Bayes' theorem:

$p(\theta \mid$ data,$I)=p(\theta \mid I) \times \frac{p(\text { data } \mid \theta, I)}{p(\text { data } \mid I)}$

where:

- $\theta$ is a set of parameter values (i.e. a hypothesis).

- data is the dataset.

- I represents information about the ensemble of hypotheses considered i.e. the type of model used and knowledge about the other models. For the remainder of this section $I$ will be implicit in likelihood expressions.

- $p($ data $\mid I)$ is a prior for the type of model used.

- $p(\theta \mid I)$ is the combined prior for the parameters.

An excellent description of the theory on which the present algorithm is based is given in Gregory \& Loredo (1992). In the present paper we will give a brief outline of the calculations, detailing only those aspects in which our work differs from the discussion of Gregory \& Loredo (1992). As a starting point we constructed an algorithm following exactly the GL prescription, and we tested it on sets of 10 simulated light curves containing transits with varying characteristics. This benchmark was later used to ensure that the modifications in our algorithm were indeed improvements.

The GL algorithm employs a family of stepwise models to describe the periodic signal plus background. Each member of the family resembles a histogram, with $m$ equal sized bins per period $P$. The family members are distinguished by the value of $m$ which is varied in the range from 2 to some upper limit (typically 15 for X-ray pulsar detection work). Such a model is capable of approximating a light curve of essentially arbitrary shape, which is desirable for detecting periodic signals of unknown shape, in contrast to the current planetary transit problem, for which the shape is known a priori. GL also employs a phase parameter $\phi$. If the time offset $o$ is defined as the time elapsed between the start of the first bin and the start of the data, $\phi=2 \pi(o / P)$. The parameters fitted by the GL method are then $P, m, \phi$ (the flux level in each bin of the model is marginalised over). In the case of planetary transits, it is not desirable to let the model vary outside the transit. We therefore have a slightly different type of model. The number of steps in the step function is $n+1$. Bin 0 is the "out of transit" bin and lasts for a large fraction of any given period, and bins 1 to $n$ are "in transit", each lasting $d / n$ where $d$ is the duration of the model transit. We have also adopted a different definition for the time offset, as we have a significant event - the transit - which we can use to determine the start of a new period. Defining the time offset $o$ as the time from the start of the data to the start of the next transit, the phase is then related to the offset in the same way as before. The parameters required are now $P, \phi$ and $d$. These parameter definitions are illustrated for both methods in Fig. 1.

As there is no feature in a step function of unconstrained shape with equal duration steps which can mark the beginning of a period in the data, the concept of phase is not well defined. Any bin in the step function could be the first. Thus we do not expect the GL method to enable phase determination directly. Only after reconstruction of the entire light curve could the position of the transit be pin-pointed relative to the start of the data. By introducing a transit feature in the model, the phase is built into the model function and we expect it to be detected effectively by the modified algorithm.

Models with a lower number $n$ of "in transit" bins will incur a lower Occam penalty factor, as emphasised in Gregory \& Loredo (1992). In general, $n$ should be chosen to be the lowest value possible. For pure detection purposes, given that transits are relatively simple events, $n=1$ should suffice. For transit reconstruction purposes, a higher value can be used.

Despite the modifications we made to the GL models, we followed the method outlined in Gregory (1999) to calculate the likelihoods.

\subsection{Likelihood calculation}

The likelihood is initially calculated for a given set of parameters $P$ (period), $d$ (duration), $o$ (offset). For convenience the results were sometimes expressed in terms of posital phase: $\mathrm{ph}=\phi / 2 \pi=o / P$.

Due to the different type of model function used, Eq. (6) in Gregory (1999), which describes the assigmnent of a bin number $j$ to each data point $y_{i}$ taken at time $t_{i}$, was replaced by the following:

$j\left(t_{i}\right)=\left\{\begin{array}{l}t_{\mathrm{mod}}: \text { if } 0<t_{\mathrm{mod}} \leq n \\ 0: \text { otherwise }\end{array}\right.$ 

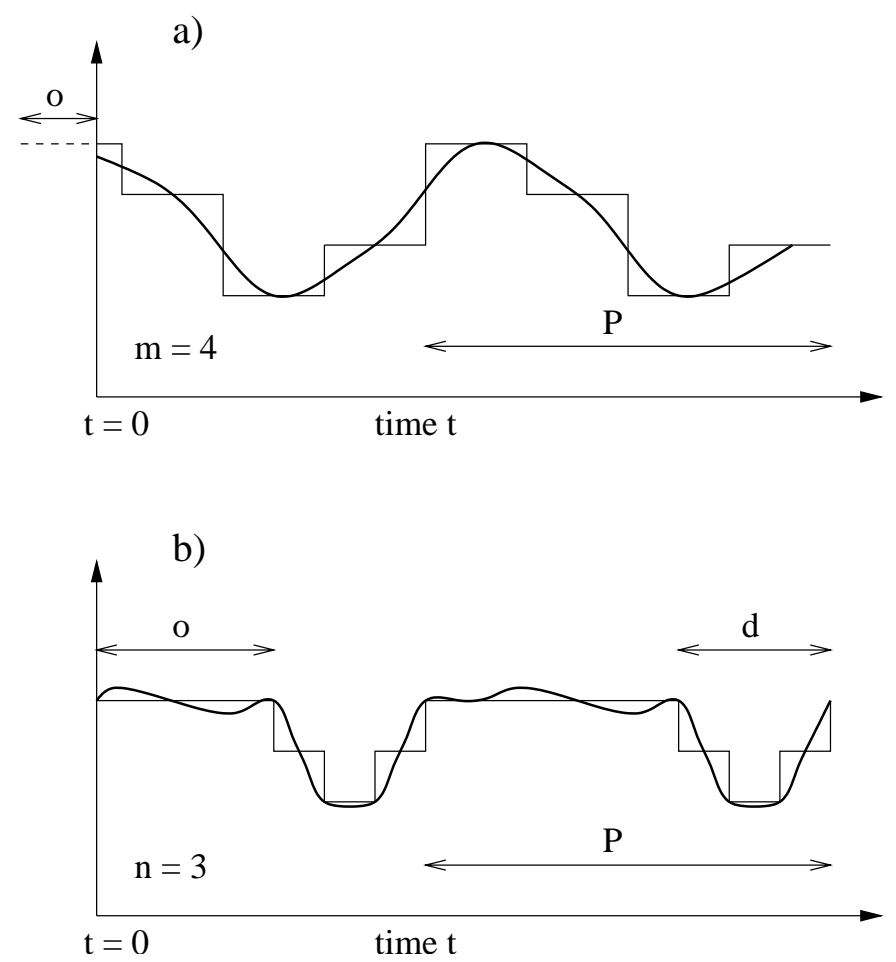

Fig. 1. Schematic illustration of the type of model and parameters used. a) GL method with $m=4$. b) Modified method with $n=3$.

where:

$t_{\mathrm{mod}}=\operatorname{int}\left(\frac{\left(t_{i}+P-o\right) \bmod (P)}{d / n}+1\right)$

$n$ is the number of bins per transit, $\operatorname{int}(x)$ is the nearest integer lower than or equal to $x$ and $(a) \bmod (b)$ is the remainder of $a$ divided by $b$.

At time $t_{i}$, the observed flux count $y_{i}$ can be written as $y_{i}=y\left(t_{i}\right)+e_{i}$ where $y\left(t_{i}\right)$ is the value predicted by the model for time $t_{i}$ and $e_{i}$ is a noise component. The noise is assumed to have a Gaussian distribution (see Gregory 1999 and references therein) with variance $\sigma_{i}^{2}$. In the present case it is appropriate and clearer to use the same value of $\sigma$ for all data points ${ }^{1}$. Strictly speaking the noise in the Eddington case is Poisson distributed (being photon shot noise), however given the large number of photons in each time bin used for the transit search this is indistinguishable from a Gaussian noise distribution. The likelihood is therefore given by:

$p($ data $\mid P, d, o)=\prod_{i=1}^{N}\left[\frac{\sigma^{-1}}{\sqrt{2 \pi}} \times \exp \left[-\frac{\left(y_{i}-y\left(t_{i}\right)\right)^{2}}{2 \sigma^{2}}\right]\right]$

where $N$ is the total number of data points.

Re-expressed in terms of the $n+1$ bins of the model:

$$
\begin{aligned}
p(\text { data } \mid P, d, o)= & \sigma^{-N}(2 \pi)^{-N / 2} \\
& \times \prod_{j=0}^{n} \exp \left[-\left(\sum_{i=1}^{n_{j}} y_{i}-r_{j}\right)^{2} / 2 \sigma^{2}\right]
\end{aligned}
$$

\footnotetext{
${ }^{1}$ In Gregory (1999) a noise parameter $b$ is introduced to account for incomplete knowledge of $\sigma$, and is then marginalised over. We have not made use of this parameter in this work.
}

where $n_{j}$ is the number of data points in bin $j$ and $r_{j}$ is the model value in bin $j$.

As shown in Gregory (1999) the argument of the exponential can be reduced to:

$$
\frac{\left(\sum_{i=1}^{n_{j}} y_{i}-r_{j}\right)^{2}}{2 \sigma^{2}}=\frac{W_{j}\left(r_{j}-\overline{d_{W_{j}}}\right)^{2}+\chi_{W_{j}}^{2}}{2} .
$$

This allows the marginalization over the $r_{j}$ 's to be performed, which we do identically to Gregory, to obtain:

$$
\begin{aligned}
p(\text { data } \mid P, d, o) & =\sigma^{-N}(2 \pi)^{-N / 2}\left(\Delta_{r}\right)^{-(n+1)}\left(\frac{\pi}{2}\right)^{(n+1) / 2} \\
& \times \exp \left[-\sum_{j=0}^{n} \chi_{W_{j}}^{2} / 2\right] \\
& \times \prod_{j=0}^{n} W_{j}^{1 / 2}\left[\operatorname{erfc}\left(y_{j, \min }\right)-\operatorname{erfc}\left(y_{j, \max }\right)\right]
\end{aligned}
$$

where:

- $\Delta_{r}=r_{\max }-r_{\min }$ is the range of values the model step function is allowed to take;

- the quantities $W_{j}, \chi_{W_{j}}^{2}, y_{j, \min }$ and $y_{j, \max }$ are taken directly from Eqs. (11) to (16) in Gregory (1999);

- $\operatorname{erfc}(y)$ is the complementary error function.

\subsection{Odds ratio calculation}

In order to use the likelihood to determine a given parameter all the other parameters must be marginalized, by multiplying by the corresponding prior and integrating over the parameter's range of values. When marginalizing the phase, in order to minimise the computing time, we incremented the phase by steps of $\pi d / P$ where $P$ is the period and $d$ the duration of the transit, corresponding to time offset increments of $d / 2$. We used the same priors for each parameter as Gregory, with a flat prior for the new parameter $d$. Although we worked in terms of period rather than frequency this does not change the calculations.

Odds ratios $O_{\mathrm{P}, \mathrm{c}}$ were then computed by comparing the probabilities as defined in Eq. (7) for a range of period values, integrating out all other parameters, to the probability obtained with a constant model denoted by the subscript "c". These odds ratios can then used to check for evidence of a periodic signal over the entire frequency range before proceeding to determine individual parameters, as described in Gregory (1999). The a posteriori probabilities needed for parameter estimation can be directly evaluated from the odds ratios by multiplying by the relevant prior and normalizing.

In Gregory \& Loredo (1992), a global odds ratio is calculated for each light curve by marginalising over all the parameters, in order to determine whether there is evidence for a periodic signal. If the global odds ratio is larger than 1 , the answer is yes. In that case, posterior probability distributions for individual parameters are used to determine the optimal parameter values. 


\subsection{Weighting factor to compensate for uneven distribution into the bins}

When the number of periods is low such that one bin might be represented four times while another only three times, or if there are gaps in the data which may not be evenly distributed over the bins, Gregory \& Loredo (1992) noted that some of their initial assumptions may fail, leading to the appearance of an erroneous trend in the posterior probability for the period.

In an appendix to Gregory \& Loredo (1992), a solution to this problem was proposed. A weighting factor $s_{j}$ is applied to each bin:

$s_{j}=\left(\frac{n_{j} m}{N}\right)^{-n_{j}}$.

This factor is derived in the context of Poisson statistics and does not apply to the present, Gaussian noise case.

Despite the low number of periods in our light curves it was found that no weighting factor was required in the benchmark algorithm that reproduced the GL identically. However it is clear that the problem is more acute in the modified algorithm. The "out of transit" bin contains many more data points than the others, and therefore has a much larger effective weight. A weighting factor is required to compensate for this problem. The expression given above for $s_{j}$ is only appropriate in the photon count context in which it was derived, not in the Gaussian noise case adopted here. A different weighting factor can be heuristically derived by considering Eq. (6). The contribution of each model level to the likelihood is a $\chi^{2}$ sum. The variance of a $\chi^{2}$ distribution is given by the number of degrees of freedom $v$. In each bin there are $n_{j}$ data points and $n_{\text {param }}$ parameters to adjust. As $n_{j} \gg n_{\text {param }}, v=n_{j}-n_{\text {param }} \simeq n_{j}$. Weighting each bin by a factor $1 / n_{j}$ is therefore equivalent to weighting by the variance. In practice this is achieved by maintaining the expressions for $\overline{d_{W_{j}}}$ and $\overline{d_{W_{j}}^{2}}$, given in Gregory (1999) in terms of $d_{i}$ and $\sigma$, but replacing $W_{j}$ by $W_{j} / n_{j}$.

This modification was implemented in our algorithm and found to give more robust results.

\subsection{Minimizing the computing time}

For a given set of parameters, the calculation of the likelihood involves summing over each element in each bin. The time required to compute the likelihood for a given set of $P, d, o$ therefore scales linearly with the number of points in the light curve. It also increases with the number of bins, but this is a slow increase. It does not depend on the individual parameter values.

The overall computing time also depends, of course, on how tightly the parameter space is sampled. It is necessary to minimise the number of trial values for each parameter without missing potentially localised likelihood maxima. Because of the relative sharpness of the peak in the posterior probability for the period, the period increment needs to be kept fairly small (typically once or twice the time step between data points). Attention was therefore concentrated on what increment was suitable in terms of phase. The results are not significantly worsened by increasing the posital phase increment from $1 / P$ (i.e. shifting the model by 1 sampling time at each increment) to $d / 2 n P$ (i.e. shifting the model by half the duration of an in-transit bin at each increment). Further increase leads to sharp steps in the posterior probability distribution (analogous to Shannon's sampling theorem).

However, the computing time is inversely proportional to the increment, and the steps in the distribution are effectively removed by dividing it by the equivalent distribution for an entirely flat light curve with the same duration, sampling and data gaps as the light curve. We call this dividing function the "window function" . We therefore used a phase increment of $d / 2 P$ and performed the division before analyzing the results. As the window function only needs to be calculated once per set of parameters, this is much faster than using a smaller increment (see Sect. 5).

Note that due to the use of this window function one should not strictly speaking use the word "posterior probability" when talking about the output of the algorithm. In the rest of this paper we will refer to "modified posterior probability" to mean "posterior probability distribution divided by the window function". This also implies that the global odds ratios mentioned in Sect. 3.3 cannot be used to directly measure the ratio of the probabilities for a periodic model compared to a constant model. Instead, we use bootstrap simulations (see Sect. 5.1) to set a threshold value of the detection statistic above which a detection is accepted.

\section{Comparing the modified algorithm with the original version}

In order to establish a reference point and to have a preliminary estimate of the modified algorithm's performance, some tests were run on both the original and the modified version. Table 1 summarizes the names and meanings of the various parameters in each method.

\subsection{A few qualitative tests}

From a typical light curve described below a number of parameters were varied one by one and the odds ratios were plotted as a function of period and as a function of phase. The base light curve lasted $11500 \times 15 \mathrm{~min}$ (119.8 days), contained a transiting giant planet with a period of 30 days, a duration of 15 hours and a posital phase (i.e. phase in radians divided by $2 \pi$ ) of 0.25 . The magnitude of the parent star was 10.0, which for Eddington corresponds to a signal to noise ratio of roughly 1400 over $15 \mathrm{~min}$, so that the depth of the transit for the Jupiter-sized planet is 14 times the noise standard deviation. It was analyzed with $m=10$ in the case of the GL method, and $n=4$ in the case of our method ${ }^{3}$. In order to sample the transit as well with the GL method as with the modified method, a much higher value of $m$ would need to be used, but this would be too computationally expensive. Instead the values of $m$ and $n$ we chosen such

\footnotetext{
2 This also has the advantage of ironing out any residual effects of the uneven bin duration not removed by the weighting factor.

3 The possibility of using $n=1$ for detection only purposes, then a larger value of $n$ for transit reconstruction, will be the subject of investigations in a further paper.
} 

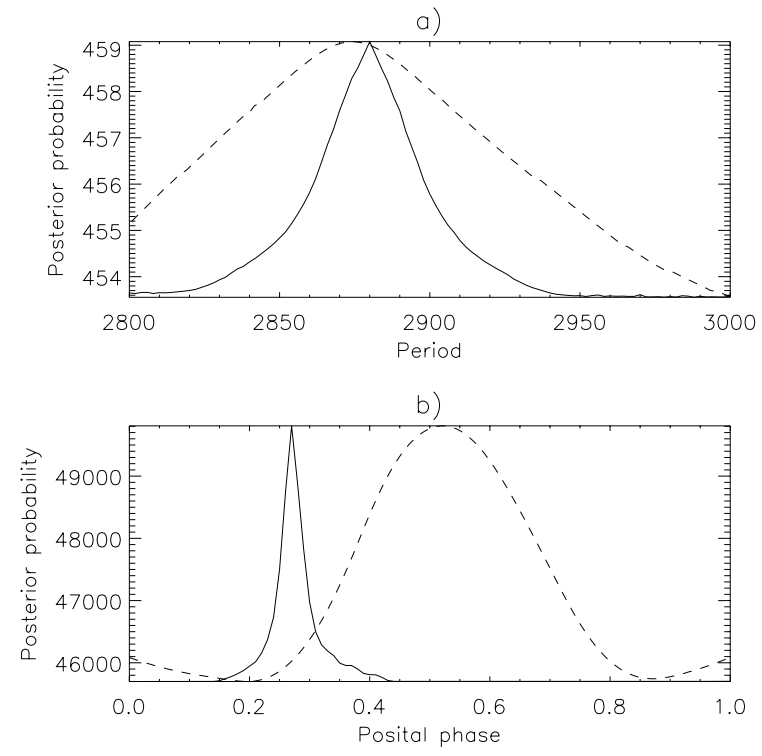

Fig. 2. Comparison of the Gregory-Loredo (GL) and modified methods for the case of Jovian planet transiting accross a 10th magnitude star (as described in Sect. 4.1), with a period of $2800 \times 15$ min a) and a phase of $0.25 \mathrm{~b}$ ). Solid line: modified algorithm, dashed line: GL method. Both methods successfully detect the period of the transits although the peak is sharper with the modified method. The GL method is unsuccessful in the phase domain (the GL phase results are folded over the 10 bins). Note that the probabilities are in arbitrary units.

that the computing times were similar. The results obtained for this benchmark case are shown in Fig. 2.

Each of the parameters (be they associated with the light curve or with the model) was varied over a small range of representative values. These one-off tests on a small parameter space confirmed some expected trends:

- for a given light curve duration the detection is less precise for longer periods as the light curve contains less transits;

- as expected, the unmodified GL method is not well suited to detecting the phase as there is no way of labeling one bin the first one. A detection is still possible by folding the posterior probability for the phase over the number of bins used. On the other hand the phase is very successfully recovered with the modified version, and the precision does not vary with the phase itself;

- the larger the value of $m$ (GL method), the sharper the detection. However $m=10$ appeared sufficient for our purposes;

- increasing the value of $n$ (modified method) does not necessarily improve the detection ability since one starts to fit the noise inside the transits, which is not periodic. When fitting Gaussian profiles it is standard to require a minimum of 2 bins per FWHM. The shape of the transit is not Gaussian but it is relatively simple, hence we multiplied by a safety factor of 2, leading to $n=4$ in further calculations. However when dealing with a particular value of $d$ it is advantageous to choose $n$ so $d$ is a multiple of it to avoid
Table 1. List of all parameters for the two types of models tested, with the symbols used to refer to them.

\begin{tabular}{lll}
\hline \hline Symbol & Method & Meaning \\
\hline$P$ & both & Period of transits \\
ph & both & Posital [0-1] phase of the transits \\
$m$ & GL & Number of steps per period \\
$d$ & modified & Duration of each transit \\
$n$ & modified & Number of steps inside each transit \\
\hline
\end{tabular}

introducing extra noise by splitting individual data points across bin boundaries (see footnote 3);

- although the modified method should in principle allow us to determine the duration of the transit, in practice this is not successful. The program may be fitting a much wider region than the transit itself. In the GL method, as there are only 10 to 20 bins per period, with $P$ of order several hundred sampling times or more, the bin in which the transit falls is much larger than the transit itself. We have seen that the loss of information this implies does not prevent the detection of the period by the GL method. The modified algorithm is likely to overestimate the transit duration because fitting a region larger than the transit does not significantly reduce the likelihood. For now the duration of the transit was simply marginalized over; once the presence of a transit is asserted and its period known, phase folding should allow a fairly quick determination of the shape and duration;

- for a given set of parameters, with $m=10$ and $n=4$, such that both algorithms have similar computing times, the detection peaks are much sharper with the modified version.

\section{Performance of the algorithm}

\subsection{Method}

To evaluate the performance of the algorithm, we used the same method as Doyle et al. (2000). For each set of trial parameters the algorithm was run first on a set of one hundred simulated light curves containing only Gaussian noise and no transits. Subsequently it was run on another set of one hundred simulated light curves containing Jovian-type planetary transits with the characteristics described in Sect. 2.1, with the same level but different realizations of the photon noise, and with uniformly distributed random phases

For each simulation, the modified posterior probabilities were plotted versus period and the value of the maximum was noted. This maximum is our "detection statistic", on the basis of which we determine whether there is a transit or not. We then plot a histogram of the detection statistics measured from running the algorithm over all the light curves with transits and one histogram for all the light curves with noise only. In other words, one histogram corresponds to the cases where the transit hypothesis is correct and one to the cases where the null hypothesis is correct. Ideally, the two distributions would be completely separated, with no overlap, and choosing a detection threshold located between the two histograms would 


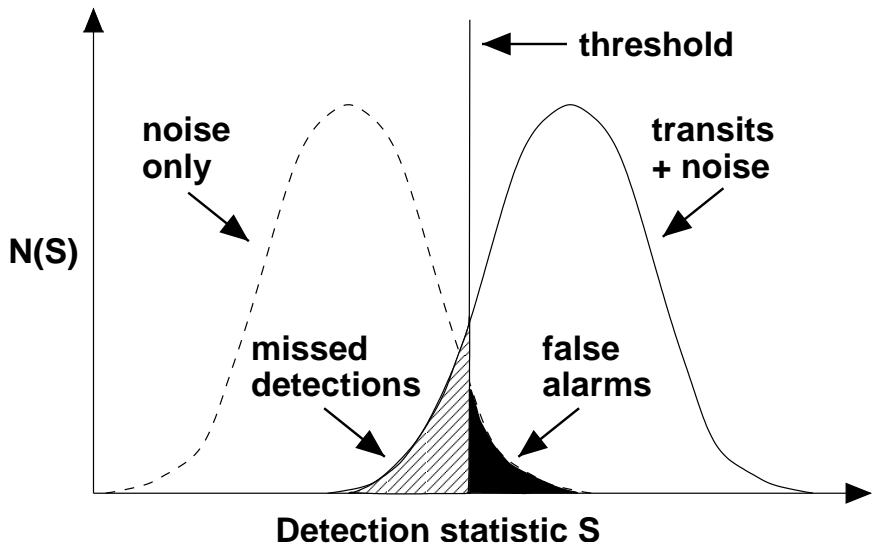

Fig. 3. Schematic diagram of the method used to set the optimal threshold and compute the false alarm and missed detection rate. Solid line: distribution of the detection statistics obtained for lightcurves with noise + transit. Dashed line: distribution of the detection statistics obtained for lightcurves with noise only. Vertical solid line: threshold. The hashed area, to the left of the threshold but under the "transits" distribution, corresponds to the missed detection rate. The filled area, to the right of the threshold but under the "noise only" distribution, corresponds to the false alarm rate.

guarantee a $100 \%$ detection rate and a $0 \%$ false alarm rate. In practice, for the cases of real interest, close to the noise level, the two histograms will show an overlap. A compromise has to be found by choosing a threshold which minimises a penalty factor designed to take into account both false alarm and missed detection rate. This is illustrated in Fig. 3.

Depending on the circumstances, it may be more important to minimise the false alarm rate than the missed detection rate. This is the approach followed by Jenkins et al. (2002), on the basis that detections from space experiments are hard to followup from the ground. An alternative view is any real transit that is rejected is a loss of valuable scientific information. As long as the false alarm rate is kept to a manageable level, further analysis of the light curves will prune out the false events. We have opted here for an intermediate position, and our penalty factor is simply the sum of the missed detection rate $N_{\mathrm{MD}}$ and the false alarm rate $N_{\mathrm{FA}}$ :

$F_{\text {penalty }}=N_{\mathrm{FA}}+N_{\mathrm{MD}}$

However, the marginalised detection algorithm yields modified posterior probabilities as a function of period, and also as a function of phase. The simultaneous use of the two detection statistics $S_{\text {per }}$ and $S_{\text {ph }}$ (plotting 2-D rather than 1-D distributions) increases the discriminating power of the algorithm, (as long as the two distributions do not have secondary maxima in 2-D space). This is shown when comparing the false alarm and missed detection rates obtained from period and phase information separately and together. The threshold in the 2-D case takes the form of a line: $S_{\mathrm{ph}}=a+b \times S_{\mathrm{per}}$. Here the optimal values of $a$ and $b$ were found by trial and error, although standard discriminant analysis techniques can be used to determine them automatically.

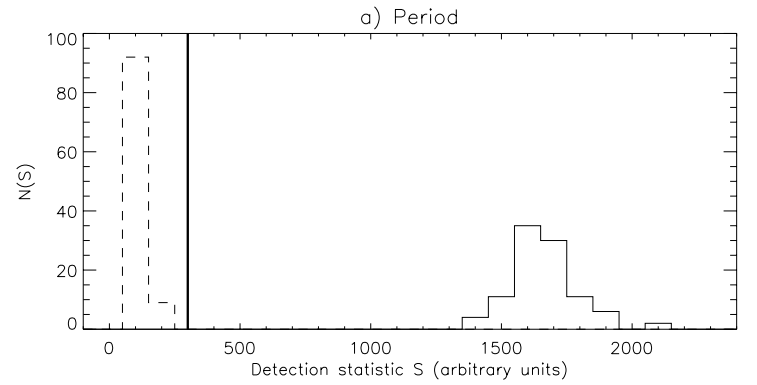

b) Phase

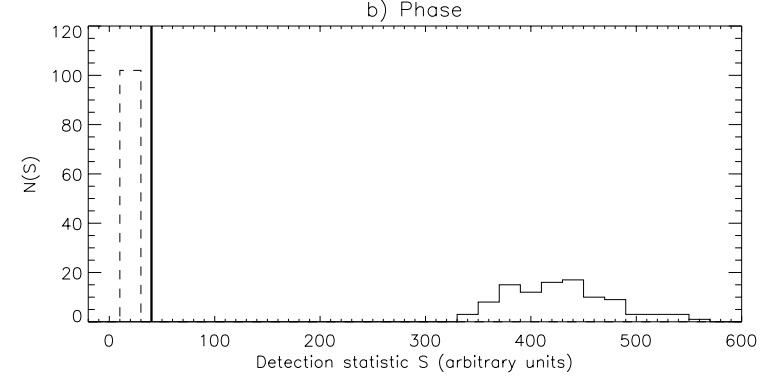

Fig. 4. Distributions of the detection statistics for an Earth-sized planet orbiting a $V=13$ star with period $P=932 \times 15 \mathrm{~min}$. Solid line: lightcurves with noise + transit. Dashed line: lightcurves with noise only. Vertical solid line: threshold value. a) Period. b) Phase. Over 100 realizations there were no false alarms and no missed detections.

\subsection{Results}

\subsubsection{An ideal case}

In Defaÿ et al. (2001a), analysis performed on the basis of 200 bootstrap samples for the COROT observations of a star with magnitude 13 and an Earth-sized planet showed, with 6 transits lasting $5 \mathrm{hr}$ each, a probability of true detection of around 0.3. We performed the simulations described in Sect. 5.1 for a similar case: Earth-sized planet orbiting a K5V type star with $V=13$ with a period of $932 \times 15 \mathrm{~min}$ and a transit duration of $5 \mathrm{hr}$. The light curve is sampled with $15 \mathrm{~min}$ bins. The noise is different from the COROT case, as we concentrate uniquely on the photon noise expected for Eddington.

The results are shown in Fig. 4 for period and phase separately. As the distributions for the noise only and transit light curves are completely separated, each parameter alone is sufficient to determine a threshold ensuring null false alarm and missed detection rates.

\subsubsection{Performance of the algorithm at the noise limit}

Given that the key scientific goal of Eddington in the field of planet-finding is the detection of habitable planets, the performance of the algorithm was extensively tested for habitable planets at (or close to) the noise limit of Eddington. The case of an Earth-like planet orbiting a $\mathrm{K}$ dwarf in a habitable orbit was used as benchmark. The light curve was simulated for a system with the following parameters:

- the star is a $\mathrm{K} 5$ dwarf $\left(R_{*}=0.8 R_{\odot}\right)$ with a range of apparent $V$-band magnitudes $V=14.0,14.5,15.0$; 
- planet with radius $R_{\mathrm{p}}=R_{\oplus}$ and a period of 4 months, orbiting the star at a distance of 0.64 A.U. (leading to a transit duration of $\approx 10.5$ hours);

- light curve duration of 16 months, containing 4 transits. The light curves were sampled every hour.

An example of a light curve is shown in Fig. 5. The resulting transit event has a depth $\Delta F / F=1.4 \times 10^{-4}$. For the Eddington baseline collecting area a star at $V=14$ will result in a photon count of $1.8 \times 10^{8}$ per hour, so that the Poisson noise standard deviation will be $1.34 \times 10^{4}$. The $S / N$ of the transit event in each 1 hour bin will thus be 1.88 . Following the same reasoning for the $V=15$ case, the $S / N$ of the of transit event in a single one hour bin is 1.19 . As there are 4 transits lasting 10 hours each in the light curves considered, the overall transit signal has a $S / N$ of $\sqrt{40} \times 1.19 \simeq 7.5$.

With the results of the simulations, an example of which is shown in Fig. 6, the analysis described in Sect. 5.1 was performed for all three magnitudes, confirming that the combined use of the two statistics improves the results. This is illustrated for the $V=14.5$ case in Figs. 7, 8 (for this particular case 1000 rather than 100 runs were computed to improve precision).
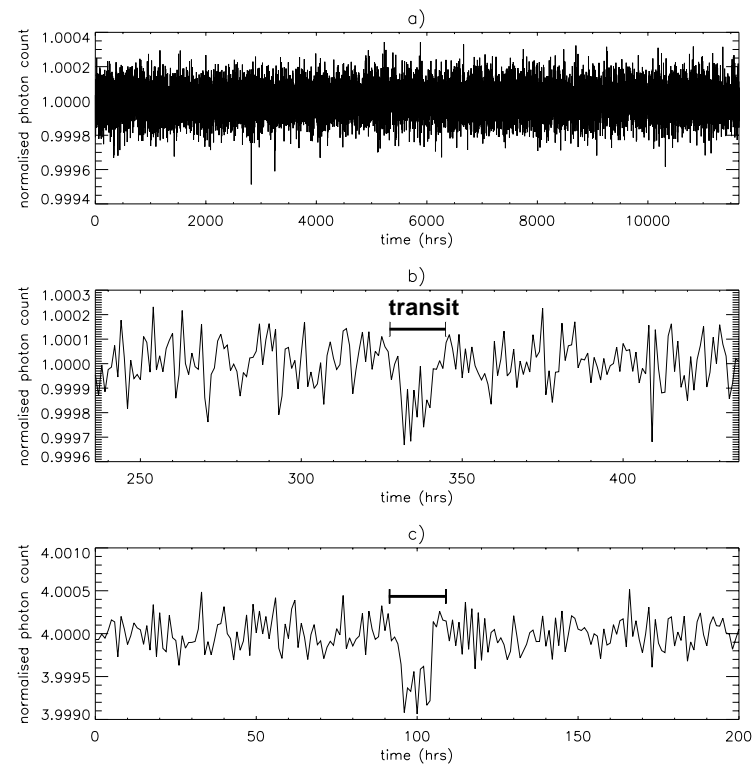

Fig. 5. An example light curve containing 4 transits of an Earthlike planet orbiting a K5V star with $V=14.5$. a) Full light curve. b) Portion around a transit. c) The four transits phase-folded.

As illustrated in Fig. 9, a mean error rate $^{4}$ of $<3 \%$ can be achieved up to magnitude 14.5. This magnitude is therefore taken as the performance limit for the algorithm for an Earthsized planet around a K5V-type star. However this analysis is not complete enough to allow a precise determination of the limit, as the noise treatment is incomplete (photon noise only being considered) and one would need more runs per simulations to compute meaningful errors on the false alarm and

\footnotetext{
4 i.e. the mean of the false alarm and missed detection rates.
}

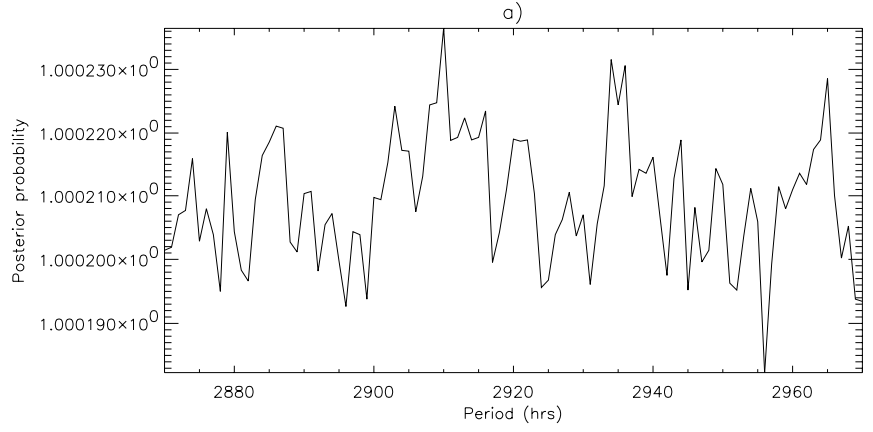

b)

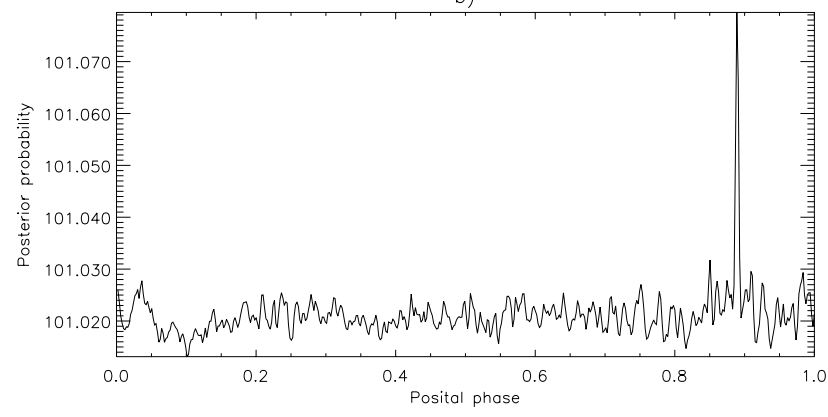

Fig. 6. Example of posterior probability distributions arising from the lightcurve shown in Fig. 5 (arbitrary units). a) Period: real value $=$ 2912 hours, error $=-2$ hours. b) Phase: real value $=0.885$, error $=$ 0.005 .

missed detection rates (sets of 1000 runs, as was done for the limiting $V=14.5$ case, should be computed for all cases).

The asymmetric shape of the distributions shown in Figs. 4, 7 and 8 implies that, even though the thresholds are chosen to minimise false alarms and missed detections equally, the optimal threshold results in more false alarms than missed detections. This could easily be avoided, if needed, by replacing Eq. (9) by:

$F_{\text {penalty }}=A \times N_{\mathrm{FA}}+N_{\mathrm{MD}}$

where $A$ is a factor greater than 1 . Alternatively one could keep the penalty factor unchanged but set a strict requirement on the maximum acceptable false alarm rate.

As in any unbiased search for periodicity in a time-series, the inclusion of a larger range of periods in the search will lead to a higher chance of finding a spurious (noise-induced) period signal in the data. The simulations used here to assess the algorithm's performance are based on a search through a relatively small range of periods. In practice, lacking any a priori knowledge of the possible periodicity of planetary orbits around the star being observed, one will have to test a large range of periods, ranging from few days (the physical limit of the period of planetary orbits) all the way to the duration of the data set (searching for individual transit events).

\subsubsection{Data gaps}

Any realistic data set will suffer from gaps in the data. While the orbits of both Eddington and Kepler have been chosen to minimize gaps, $100 \%$ availability is not realistic, and gaps 


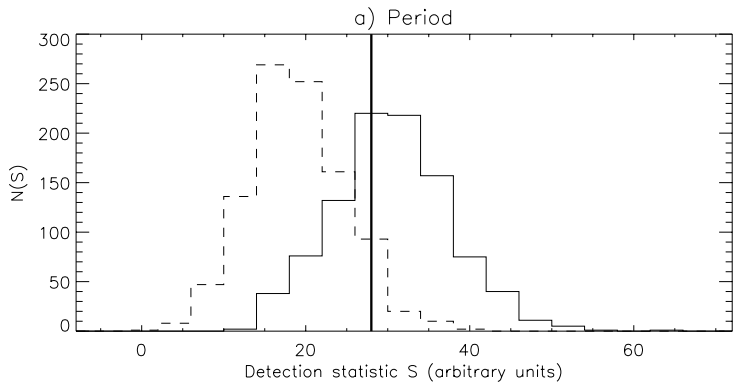

b) Phase

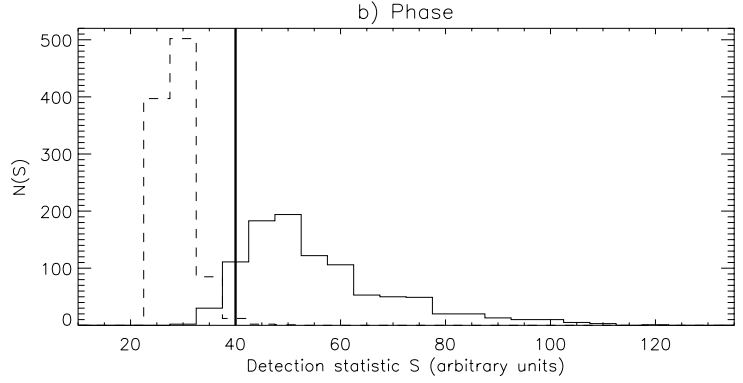

Fig. 7. Distributions of the detection statistics for an Earth-sized planet orbiting a $V=14.5$ star with period $P=4$ months. Solid line: lightcurves with noise + transit. Dashed line: lightcurves with noise only. Vertical solid line: threshold value. a) Period: 190 false alarms and 185 missed detections over 1000 realizations. b) Phase: 27 false alarms and 14 missed detections over 1000 realizations.

will be present due to e.g. telemetry dropouts, spacecraft momentum dumping maneuvers, showers of solar protons during large solar flares, etc. For this reason any realistic algorithm must be robust against the presence gaps in the data, showing graceful degradation as a function of the fraction of data missing from the time series.

We have therefore tested the algorithm discussed here using simulated light curves with 5\%, 10\% and $20 \%$ data gaps, randomly distributed in the data, i.e. $5 \%$ of the points in the time series are selected randomly with a uniform distribution and removed from the light curve. The gaps will probably not be randomly distributed in reality, but as the typical gap duration is expected to be of order 1 or 2 hours, simulated random gaps can already be used to test the algorithm's robustness. For reasons of computing time, to avoid having to recalculate the "window function" at each run, the distribution of the data gaps is the same for all runs of a simulation. As the gaps are chosen one by one there are rarely gaps of more than two consecutive time steps, i.e. 2 hours. Note that e.g. the Eddington mission is designed to produce light curves with a duty cycle $\geq 90 \%$, so that the case with $20 \%$ data gaps represents a worst case analysis.

The results are shown in Fig. 10. There is visibly very little degradation up to $20 \%$ data gaps. When using $S_{\text {per }}$ alone or the two statistics combined there is no perceptible difference. We can therefore say this algorithm is robust at least for data gaps of the type likely to occur due to e.g. telemetry dropouts, which last only a few hours. One would also expect the algorithm to perform well in the presence of longer gaps: the effect of gaps is to render the number of samples per bin uneven, and this is already the case for this particular method with no gaps at all.
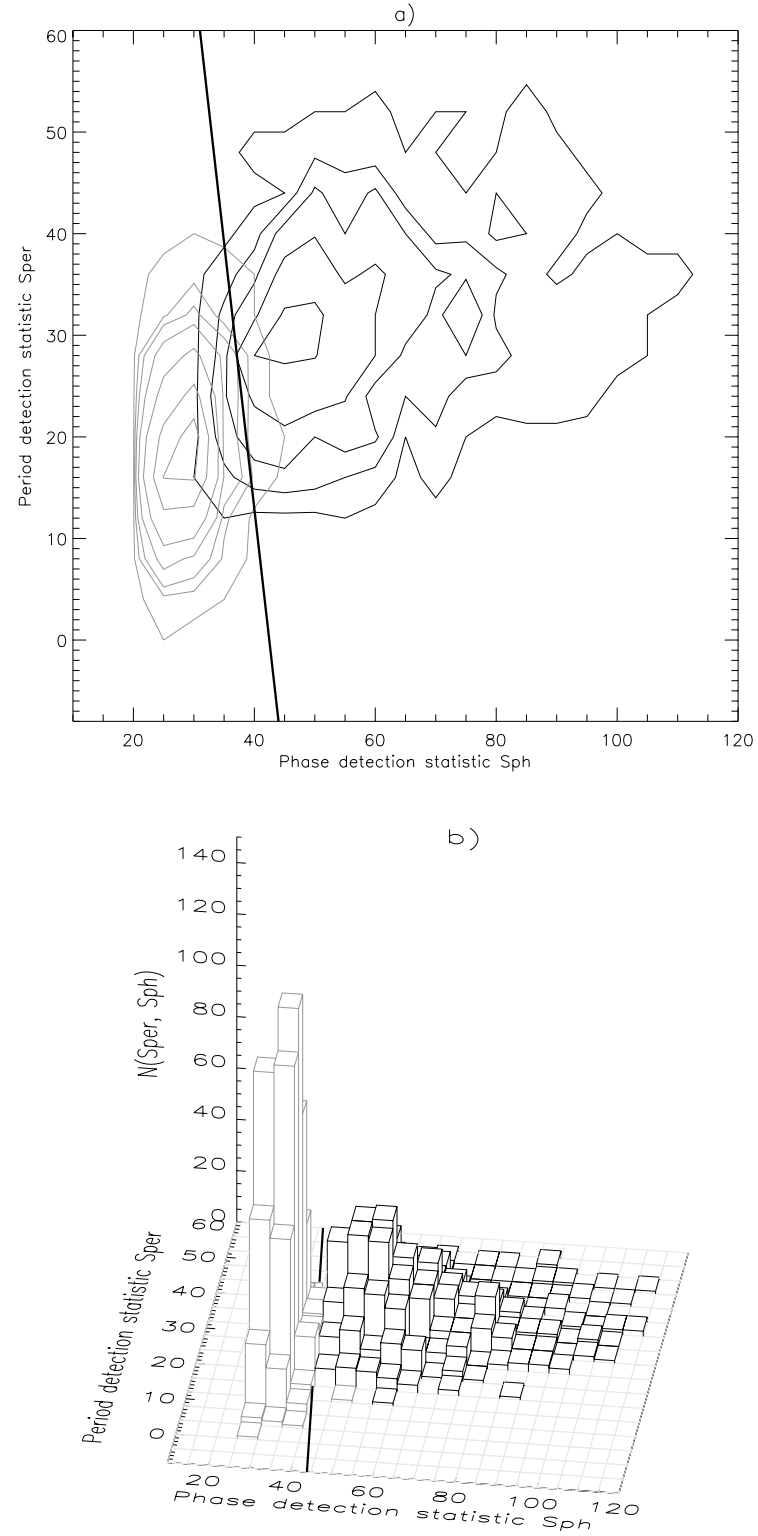

Fig. 8. a) Contour plot and b) 3-D representation of the twodimensional distributions of the period and phase detection statistics for an Earth-sized planet orbiting a $V=14.5$ star with pe$\operatorname{riod} P=4$ months. Black: lightcurves with noise + transit. Grey: lightcurves with noise only. Solid line: Optimal threshold line. $\left(S_{\mathrm{ph}}=\right.$ $42.47-1.191 \times S_{\text {per }}$ ), yielding 29 false alarms and 9 missed detections over 1000 realisations.

\subsubsection{Number of transits in the data}

The planetary transits detection phase of the Eddington mission is planned to last 3 years with a single pointing for the entire duration of that phase. There will therefore be three or four transits in the light curve for a typical habitable planet. However, other missions such as COROT are planned with shorter ( 5 months) pointings and it is of interest for this type of mission to study the degradation of the algorithm's performance as the number of transits in the light curve reduces. If the algorithm performs well with 2 or less transits, in the context of Eddington it may also allow the detection of "cool Jupiters", i.e. Jupiter-sized planets with orbits more similar to those of the 


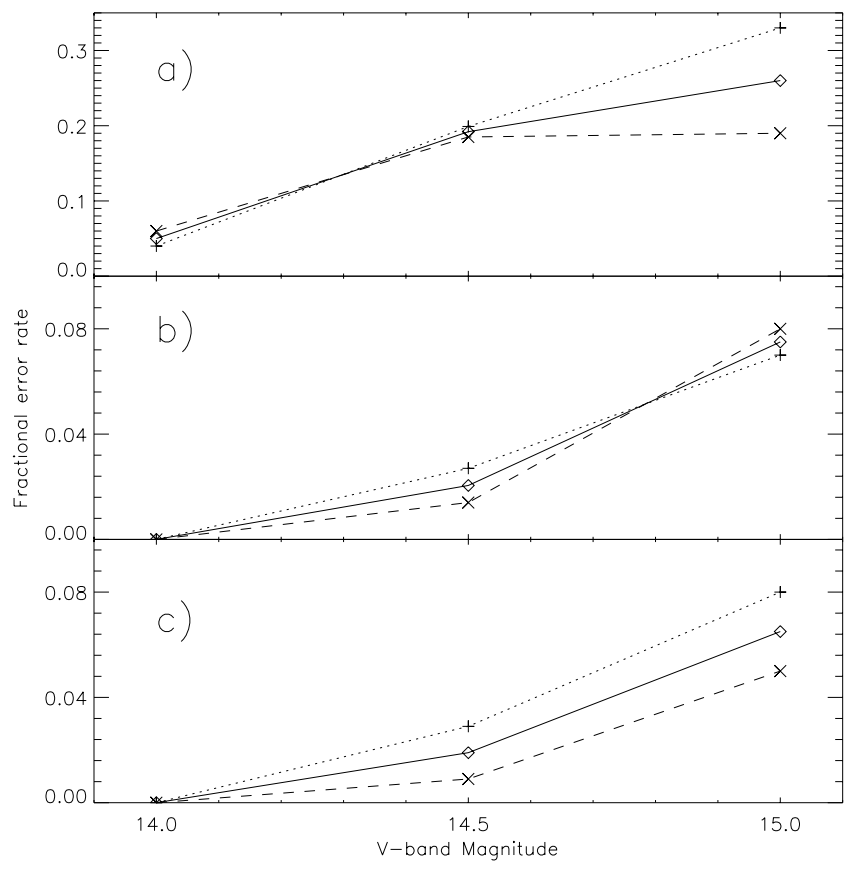

Fig. 9. Evolution of the algorithm's performance (in terms of fractional error rates) with magnitude ( $P=4$ months, $V=14.5$, Light curve duration 16 months). a) Using the period statistic only. b) Using the phase statistic only c) combining the two statistics dotted line: false alarm rate. Dashed line: Missed detection rate. Solid line: mean error rate.

gaseous giants in our solar system. This would be of relevance to the question of how typical our solar system is.

Sets of 100 runs with the characteristics specified in Sect. 5.2.2 for a star of magnitude 14.5 were computed for light curve durations of 4, 8, 12, 16 and 20 months, containing between 1 and 5 transits. The results are shown in Fig. 11. The degradation only becomes significant when less than three transits are present. However, even mono-transits could be detectable for larger planets at that magnitude.

Defaÿ et al. (2001a) compared a matched filter approach with a Bayesian method based on the decomposition of the light curve into its Fourier coefficients. Their results suggest that the performance degradation in the low number of transits case is faster for the Bayesian method than for the matched filter. This is because the matched filter makes use of assumptions about the transit shape. It is also shown that when the Bayesian method fails to detect a transit, it can still reconstruct it if the detection is performed using a matched filter. Our algorithm has not been directly compared to a matched filter. Its very design is based on the search for a short periodic signal in an otherwise flat lightcurve, which is itself an assumption about the shape of the signal. The matched filter makes use of more detailed knowledge of the transit shape and is therefore likely to perform better in the low transit number limit. However our algorithm with $n=1$ may provide already a very good approximation to the relatively simple shape that is a transit, and therefore perform nearly as well.

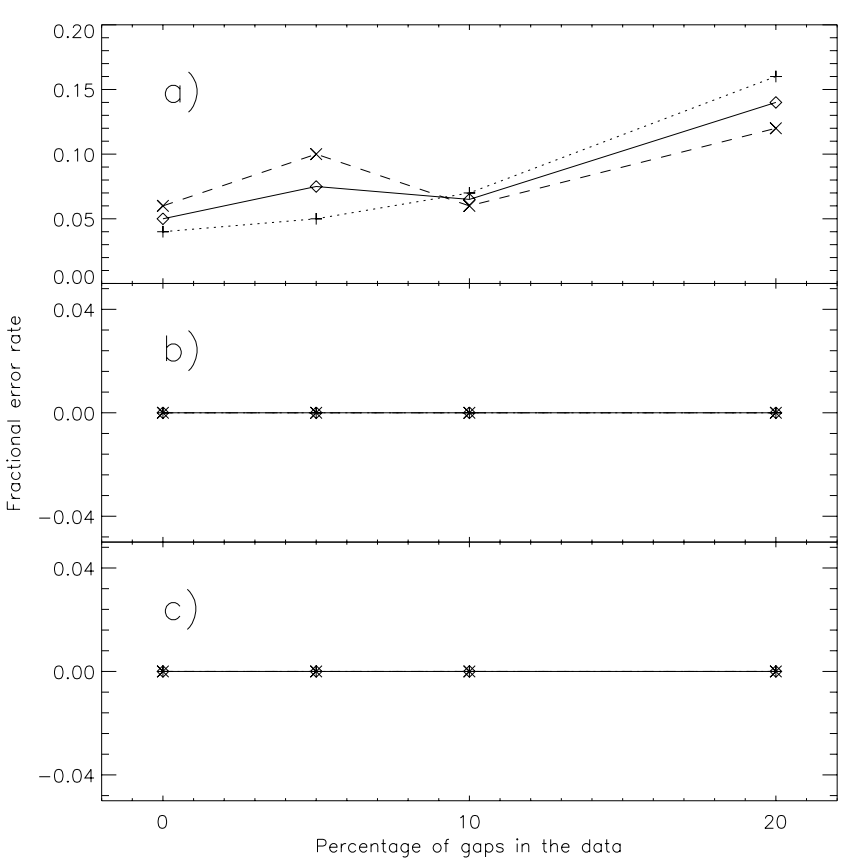

Fig. 10. Evolution of the algorithm's performance with data gaps $(P=$ 4 months, $V=14.0$, Light curve duration 16 months). a) Using the period statistic only. b) Using the phase statistic only. c) Combining the two statistics. Dotted line: false alarm rate. Dashed line: missed detection rate. Solid line: mean error rate.

\subsubsection{Differences in the two statistics}

The two a posteriori probabilities show a different behavior. In general the phase statistic is far more discriminatory than the period statistic. The period statistic's lesser effectiveness may be explained in the following way. If the phase is wrong, even if the period is right, it is likely none of the transits will be matched. If the phase is right, whatever the period, at least the first transit will be matched by the model. First we consider the likelihood distribution a function of phase, normalised over all periods. For an incorrect phase the contribution from the correct period is nil as all transits are missed, but for the correct phase all trial periods produce a non-negligible contribution (the correct period of course contributing most). The likelihood distribution as a function of phase is therefore sharply peaked. Then we consider the likelihood distribution as a function of period, normalised over all phases. The contribution from the correct phase is non-negligible whatever the period. When the period is correct, the contribution from the correct phase is washed out by the contributions from all the incorrect phases. The likelihood distribution as a function of period is therefore less sharply peaked.

However the combined use of the two parameters is more successful than the phase statistic alone. The reason for this is illustrated in Fig. 8: in 2-D space the two distributions are aligned on a diagonal, such that no single value cutoff is optimal in either direction, compared to the line shown. In an upcoming paper, the direct use of a combined statistic shall be investigated. The global odds ratio described in Sect. 3.3 could be used for such a purpose. We have noted in Sect. 3.5 that the global odds ratio for a given lightcurve cannot be used as an 


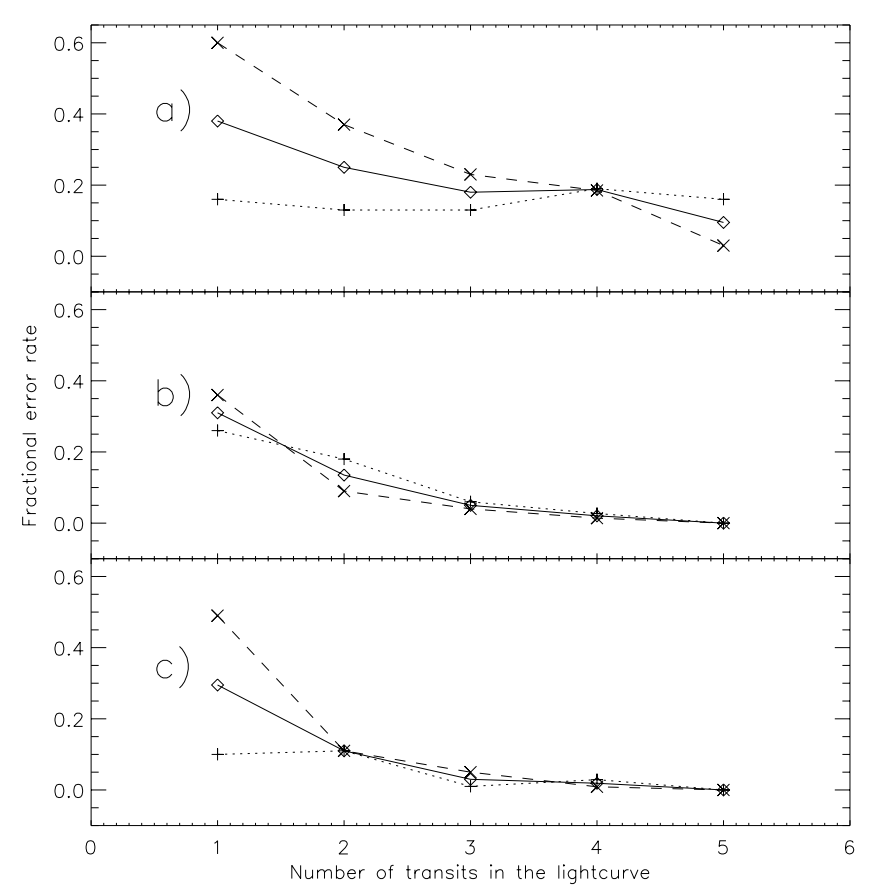

Fig. 11. Evolution of the algorithm's performance with the number of transits in the light curve, i.e. the light curve duration ( $P=4$ months, $V=14.5$ ). a) Using the period statistic only. b) Using the phase statistic only. c) Combining the two statistics. Dotted line: false alarm rate. Dashed line: missed detection rate. Solid line: mean error rate.

absolute statitstic in the context of the present method. It can however be used as relative detection statistic, like $S_{\mathrm{per}} \& S_{\mathrm{ph}}$, combined with bootstrap simulations.

\section{Discussion}

Efficient data processing is one of the challenges for the upcoming generation of large scale searches for exo-planets through photometric transits. While radial velocity searches concentrate on limited number of stars, transit searches will investigate simultaneously large numbers of stars, and produce large amounts of data (photometric light curves) for each of them. A computationally efficient and robust algorithm for the processing of these data sets is necessary to make transit searches feasible. It is likely that the photometric time series which represent the observational product of the transit searches will be analyzed in different stages, using more than a single approach. In particular, a first level of processing (after instrumental effects have been removed) should concentrate on singling out high-probability transit candidates, while efficiently pruning out the large number (more than $90 \%$, even if all stars have planets, due to the low probability of transit events) of light curves in which no transits are present. In this first stage of analysis the ability to efficiently screen real transits in the data - even at the price of a moderate number of false alarms - is a key requirement for the algorithm. The candidate light curves in which a transit is suspected will then later be subject to a more detailed processing, which can then afford to be computationally less efficient (given it has to operate on a much smaller amount of data).
The algorithm we have developed and discussed here is able to detect transit events at the limit of the photon noise present in the light curve. It shows a graceful degradation of its performance as function of different parameters of interest, e.g. the noise level in the data, as well as the presence of data gaps and the number of transits actually observed. Its strong sensitivity to the phase of periodic transits supplies significant additional information to be then used by further steps of processing for e.g. the reconstruction of the transit parameters. Thus, while little used in astronomy, Bayesian algorithms appear to be a powerful tool in the processing of transit data.

\section{Conclusions and future work}

A novel algorithm to detect transits due to extra-solar planets in stellar light curves has been developed and tested. The algorithm, based on a Bayesian approach, has proved successful in the tests performed so far, which include the effects of photon noise and data gaps. Using the photometric accuracy and throughput expected for the Eddington mission, we are able to detect an Earth-sized planet orbiting a K5V-type star with a period of 4 months down to an apparent stellar magnitude of $V \simeq 14.5$. Randomly distributed data gaps lasting up to two hours each and covering up to $20 \%$ of the light curve do not significantly affect the performance of the algorithm. The minimum number of transits in one light curve required for high confidence detections is three, however the algorithm's performance degrades gracefully for small number of transits, so that detections are possible for individual transits, albeit at a lower confidence level. This will allow for the detection of larger planets in long-period orbits (analogous to the gaseous giants of our solar system), likely to transit only once in the three year planet detection phase planned for the Eddington mission.

The most serious additional noise source to perturb planetary transit detections from space, is likely to be intrinsic stellar micro-variability (mostly activity-induced). At the moment it is also the least well investigated. The consequences of activity on the detection efficiency (using simulated light curves based on the solar light curves recorded by the VIRGO instrument on board SOHO, which spans all solar activity levels, from solar minimum to solar maximum) will be the subject of a future paper, in which the feasibility and effectiveness of using color information, as well as a number of pre-processing techniques such as whitening, will also be investigated.

The algorithm we have developed and discussed here has the potential to form part of a powerful, multi-stage approach to analysing transit lightcurves. A more optimised processing method will be discussed in a separate paper. It will include a variability filtering stage, followed by distinct detection and parameter estimation stages, using a combination of a matched filter approach and of the present algorithm.

The performance of the algorithm presented here shows that the search of planetary transits with amplitudes comparable to the intrinsic noise level of the data set is fully feasible, and thus represents an important element in the development of the future generation of transit-based planet finding missions.

Acknowledgements. This work was supported by the European Space Agency's Young Graduate Trainee program. The authors are grateful 
to Hans Deeg for making his transit modelling package UTM available. We wish to thank Mike Irwin, David Katz \& Rolf Jansen for advice, and Celine Defaÿ for advice and access to her algorithm. We are also grateful for the thorough and helpful comments of the referee.

\section{References}

Butler, R. P., Marcy, G. W., Fischer, D. A., et al. 2001, in Planetary Systems in the Universe: Observation, Formation and Evolution, ed. A. Penny, P. Artymowicz, A. M. Lagrange, \& S. Russel, IAU Symp., Vol. 202

Deeg, H. 1999, www .iac.es.galeria/hdeeg/idl_hans_lib/utm
Defaÿ, C., Barge, P., \& Deleuil, M. 2001a, A\&A, submitted

Defaÿ, C., Deleuil, M., \& Barge, P. 2001b, A\&A, 365, 330

Doyle, L. R., Deeg, H. J., Kozhevnikov, V. P., et al. 2000, ApJ, 535, 338

Favata, F., \& the Eddington Science Team. 2000, Eddington Assessment Study Report, Tech. Rep. ESA-SCI(2000)8, ESA

Gilliland, R. L., Brown, T. M., Guhathakurta, P., et al. 2000, ApJ, 545, L47

Gregory, P. C. 1999, ApJ, 520, 361

Gregory, P. C., \& Loredo, T. J. 1992, ApJ, 398, 146

Jenkins, J. M., Caldwell, D. A., \& Borucki, W. J. 2002, ApJ, 564, 495

Mayor, M., \& Queloz, D. 1995, Nature, 378, 355

Van Hamme, W. 1993, ApJ, 106, 2096 